\title{
Comparison of the benefit of primary prevention implantable cardioverter-defibrillator therapy in ischemic versus nonischemic dilated cardiomyopathy
}

\author{
Kun Wang ${ }^{1}$, Xinyue $\mathrm{Xu}^{1}$, Yu Qi ${ }^{1}$, Yihai $\mathrm{Liu}^{2}$, Lina Kang ${ }^{1}$, Xiaohong $\mathrm{Li}^{1}$, Rongfang Lan ${ }^{1}$, \\ Lian Wang ${ }^{1}$, and Wei Xu ${ }^{1}$ \\ ${ }^{1}$ Nanjing University Medical School Affiliated Nanjing Drum Tower Hospital \\ ${ }^{2}$ Nanjing Drum Tower Hospital
}

November 17, 2020

\begin{abstract}
Introduction: Ischemic cardiomyopathy (ICM) and idiopathic dilated cardiomyopathy (DCM) share common structural alterations with a high mortality from sudden cardiac death (SCD) and pump failure. Implantable cardioverter-defibrillator (ICD) has, since inclusion in international guidelines, been confirmed beneficial and cost-effective for primary prevention of SCD in patients with ICM, while huge debates in non-ischemic heart disease. This study was to compare the primary prophylactic value of ICD therapy in patients with ICM or DCM to identify a subgroup with greater advantage specially. Methods: We conducted a retrospective, single-center study, which enrolled 82 patients with ICM or DCM and guideline indications for primary prophylactic ICD or cardiac resynchronization therapy-defibrillator (CRT-D). Primary end-point was all-cause mortality and secondary outcomes included SCD and cardiovascular death. Results: During a median follow-up of 38.5 months, 78 patients baseline data were analyzable. The primary outcome occurred in 8 patients in ICM group and 5 patients in DCM group ( $\mathrm{p}=$ 0.012). Cardiovascular death occurred in 5 patients in ICM group and 3 patients in DCM group [hazard ratio (HR) $0.119,95 \%$ confidence interval (CI) 0.016-0.860, $\mathrm{P}=0.035$ ]. Resuscitated cardiac arrest or sustained ventricular tachycardia occurred in 4 patients in ICM group and 8 patients in DCM group (HR 0.294, 95\% CI 0.040-2.144, P = 0.227). Conclusions: DCM patients with ICD implantation could gain more benefit with a reduction in the risk of all-cause mortality and cardiovascular disease compared with ICM patients, while the occurrence of SCD had no difference in two groups.
\end{abstract}

Comparison of the benefit of primary prevention implantable cardioverter-defibrillator therapy in ischemic versus nonischemic dilated cardiomyopathy

Kun Wang, $\mathrm{MD}^{1 \#}$; Xinyue $\mathrm{Xu}, \mathrm{MD}^{1 \#}$; Yu Qi, $\mathrm{PhD}^{1 \#}$; Yihai Liu, $\mathrm{PhD}^{2}$; Lina Kang, $\mathrm{MD}^{1}$; Xiaohong Li, $\mathrm{MD}^{1}$; Rongfang Lan, $\mathrm{MD}^{1}$; Lian Wang, $\mathrm{MD}^{1 *}$; Wei $\mathrm{Xu}, \mathrm{MD}^{1 *}$

\# These authors contributed equally.

1)Department of Cardiology, Affiliated Nanjing Drum Tower Hospital of Nanjing University Medical School, Nanjing 210008, China.

2)Department of Cardiology, Nanjing Drum Tower Hospital, Clinical College of Nanjing Medical University, Nanjing 210008, China.

This work was supported by grants from the Key Projects of Science and Technology Development Foundation of Nanjing Department of Health (201704003 to L. Wang) and the Six Talent Peaks Project in Jiangsu Province (2016-WSN-157 to L. Wang).

\section{*Correspondence}


Wei Xu , MD, Tel: +86 13390900868, E-mail: 13390900868@163.com

Lian Wang, MD, Tel: +86 18115131666, E-mail: wanglianglyy@163.com

\section{Abstract}

Introduction:Ischemic cardiomyopathy (ICM) and idiopathic dilated cardiomyopathy (DCM) share common structural alterations with a high mortality from sudden cardiac death (SCD) and pump failure. Implantable cardioverter-defibrillator (ICD) has, since inclusion in international guidelines, been confirmed beneficial and cost-effective for primary prevention of SCD in patients with ICM, while huge debates in non-ischemic heart disease. This study was to compare the primary prophylactic value of ICD therapy in patients with ICM or DCM to identify a subgroup with greater advantage specially.

Methods: We conducted a retrospective, single-center study, which enrolled 82 patients with ICM or DCM and guideline indications for primary prophylactic ICD or cardiac resynchronization therapy-defibrillator (CRT-D). Primary end-point was all-cause mortality and secondary outcomes included SCD and cardiovascular death.

Results: During a median follow-up of 38.5 months, 78 patients baseline data were analyzable. The primary outcome occurred in 8 patients in ICM group and 5 patients in DCM group $(\mathrm{p}=0.012)$. Cardiovascular death occurred in 5 patients in ICM group and 3 patients in DCM group [hazard ratio (HR) 0.119, 95\% confidence interval (CI) 0.016-0.860, $\mathrm{P}=0.035$. Resuscitated cardiac arrest or sustained ventricular tachycardia occurred in 4 patients in ICM group and 8 patients in DCM group (HR 0.294, 95\% CI 0.040-2.144, P $=0.227)$.

Conclusions: DCM patients with ICD implantation could gain more benefit with a reduction in the risk of all-cause mortality and cardiovascular disease compared with ICM patients, while the occurrence of SCD had no difference in two groups.

\section{Key words}

Ischemic cardiomyopathy; Dilated cardiomyopathy; Sudden cardiac death; Implantable cardioverter defibrillator

\section{Introduction}

Heart failure (HF), as a final common stage of many cardiovascular diseases, is a major public health problem affecting approximately 40 million people globally. ${ }^{[1]}$ A spontaneous progressive clinical syndrome with dyspnea, water and sodium retention, pump failure underlying causes, pathophysiological complexities, and concomitant comorbidities, which made both diagnosis and treatment particularly challenging. ${ }^{[2]}$ Ischemic cardiomyopathy (ICM) and idiopathic dilated cardiomyopathy (DCM) share common structural alterations with a high mortality from sudden cardiac death (SCD) and pump failure. ${ }^{[3]}$ According to New York Heart Association (NYHA) classification, the all-cause mortality of patients with NYHA class II is $5 \%$ to $15 \%$, of which $50 \%-60 \%$ is sudden death. The all-cause mortality rate of NYHA class III increases to $30 \%$ to $50 \%$, and class IV usually exceeds $50 \%{ }^{[4]}$

During the past decades, great progresses have been made in decreasing the occurrence of SCD. In particular, ICD implantation has become a priority for primary prevention ICDs in patients with indication. ${ }^{[5]}$ However, individual DCM trials have failed to gain the conclusion of a mortality benefit with prophylactic ICD implantation. ${ }^{[6]}$ European guidelines have recommended that ICD therapy to prevent SCD in patients with symptomatic HF (NYHA class II-III) and LVEF [?]35\% after [?]3 months of optimal medical therapy who are expected to survive for at least 1 year with good functional status. ${ }^{[7,8]}$ Nevertheless, in most studies HF patients with DCM represent a minor subgroup of the overall study population. ${ }^{[9]}$ Whether there is a difference in prognosis between primary prophylactic ICD therapy in patients with ICM and DCM remains unclear. To address this issue, we conducted a retrospective study, which enrolled 82 patients with DCM or ICM and with guideline indications for primary prophylactic ICD or CRT-D treatment. We aimed to identify subgroups with greater or lesser advantage from ICD therapy. 


\section{Methods}

\section{Study population}

This study has been approved by the Ethics Committee of Nanjing Drum Tower Hospital have therefore been performed in accordance with the Declaration of Helsinki and its later amendments. We enrolled 82 patients with ICM or DCM who received ICD implantation for primary prevention between January 2010 and August 2018 admitted in the Department of Cardiology of Nanjing Drum Towel Hospital. Among them, 26 were ICM and 56 were DCM. A total of 78 patients were followed up, including 25 patients with ICM and 53 patients with DCM. Clinical characteristics, medications, 24-h Holter electrocardiograms (ECGs) were recorded on admission.

\section{Inclusion criteria}

ICM patients: 1) Patients with chronic congestive heart failure; 2) Patients with definite myocardial infarction and who have received percutaneous coronary intervention (PCI) or surgical coronary artery bypass grafting (CABG); 3) Electrocardiogram, echocardiography or cardiac magnetic resonance show large-scale necrosis of myocardium or local dyskinesia of ventricular wall; 4) After 3 months of treatment with standard anti-heart failure drugs, echocardiography confirmed left ventricular ejection fraction (LVEF) [?]35\%; 5) NYHA class II-III or IV patients considering CRT-D or NYHA class IV patients waiting for heart transplantation. ${ }^{[7]}$

DCM patients: 1) Patients with chronic congestive heart failure; 2) After 3 months of treatment with standard anti-heart failure drugs, echocardiography confirmed LVEF [?]35\%, the whole heart is enlarged, and LVEDD (left ventricular end diastolic diameter) $>55 \mathrm{~mm}$ in male or $>50 \mathrm{~mm}$ in female, or the wall motion is weakened; 3) Exclude heart enlargement and EF reduction caused by other diseases (such as hypertrophic cardiomyopathy, alcoholic cardiomyopathy, valvular disease, cardiomyopathy caused by hyperthyroidism, etc.); 4) NYHA class II-III or IV patients considering CRT-D or NYHA class IV patients waiting for heart transplantation. ${ }^{[8]}$

\section{Exclusion criteria}

1) Suffering from heart disease other than ICM and DCM which leads to chronic heart failure; 2) Patients had acute left heart failure; 3) The life expectancy of the patient is less than 1 year; 4) Patient experienced cardiovascular events such as SCD, ventricular fibrillation, cardiopulmonary resuscitation or continuous ventricular tachycardia.

\section{Outcomes}

The primary outcome was all-cause mortality. The secondary outcomes were: SCD, other cardiogenic deaths (including death due to malignant arrhythmia such as ventricular tachycardia, ventricular fibrillation, etc.) and other events (including device infection, resuscitated cardiac arrest or sustained VT, sustained VT demand cardioversion or cardioversion, heart transplantation, ICD false discharge).

\section{Statistical Analysis}

Baseline characteristics were compared using student's t test or Wilcoxon test for continuous variables or the chi-square test for categorical variables between the ICM and DCM groups. The cumulative risks of all-cause death were estimated using the Kaplan-Meier method and the use of the log-rank test. Analysis was performed with SPSS Statistics software (version 26) and the variables were presented as number or mean \pm standard deviation, as appropriate. When two-sided $\mathrm{P}$ value $<0.05$, the difference considered statistically significant.

\section{Results}

\section{Baseline Characteristics of the Patients}


Baseline characteristics of the included patients in each group were shown in Table 1 .Compared with patients in DCM group, ICM group were significantly older $(\mathrm{p}<0.001)$ and the prevalence of hypertension $\left(\mathrm{p}_{\mathrm{i}} 0.001\right)$ and diabetes $(\mathrm{p}=0.033)$ in the ICM group was significantly higher than that in DCM group. LVEF ( $p=0.002)$ were significantly higher than that of DCM patients and diastolic blood pressure (DBP, $p$ $=0.015)$ were significantly lower than that of DCM patients. Other demographic variables were comparable between the two groups. NYHA classification, B-type natriuretic peptide (BNP) and systolic blood pressure (SBP) had no statistically significant difference. More percentage of people in the DCM group received CRT $(37.7 \%$ vs. $12 \%, \mathrm{p}=0.009$; Table 1$)$. The large majority of patients in both groups received heart failure medications according to the ESC Guidelines while there was no significant difference except for P2Y12 receptor inhibitors $(\mathrm{p}<0.001)$ and statins $(\mathrm{p}=0.002)$.

Table 1. Characteristics of the Patients at Baseline.

\begin{tabular}{|c|c|c|c|}
\hline Baseline characteristics & $\operatorname{ICM}(\mathrm{n}=25)$ & $\mathrm{DCM}(\mathrm{n}=53)$ & p-value \\
\hline$\overline{\text { Age (years) }}$ & $71.72 \pm 11.18$ & $61.85 \pm 10.85$ & ¡0.001 \\
\hline Male/Female & $20 / 5$ & $43 / 10$ & 0.906 \\
\hline Month & $46.25 \pm 51.92$ & $57.06 \pm 54.35$ & 0.667 \\
\hline $\operatorname{LVEF}(\%)$ & $32.67 \pm 3.66$ & $28.43 \pm 5.92$ & 0.002 \\
\hline $\begin{array}{l}\text { NYHA Class II-III or } \\
\text { IV }\end{array}$ & $2.92 \pm 0.65$ & $2.81 \pm 0.62$ & 0.859 \\
\hline Hypertension, n (\%) & $22(88.0)$ & $21(39.6)$ & ¡0.001 \\
\hline $\mathrm{SBP}(\mathrm{mmHg})$ & $124.52 \pm 14.39$ & $122.11 \pm 17.99$ & 0.560 \\
\hline $\mathrm{DBP}(\mathrm{mmHg})$ & $70.96 \pm 9.68$ & $77.55 \pm 11.46$ & 0.015 \\
\hline Diabetes, n (\%) & $11(44.0)$ & $11(20.8)$ & 0.033 \\
\hline $\begin{array}{l}\text { Atrial fibrillation, } \mathrm{n} \\
(\%)\end{array}$ & $6(24.0)$ & $12(22.6)$ & 0.894 \\
\hline $\mathrm{CAG}, \mathrm{n}(\%)$ & $22(88.0)$ & $20(37.7)$ & ¡0.001 \\
\hline CTA, n (\%) & $0(0.0)$ & $2(3.8)$ & 0.159 \\
\hline $\mathrm{BNP}(\mathrm{Ng} / \mathrm{L})$ & $908.50 \pm 717.34$ & $891.40 \pm 733.72$ & 0.929 \\
\hline $\begin{array}{l}\text { Creatinine }(\mathrm{mmol} / \mathrm{L}) \\
\text { Medication }\end{array}$ & $93.86 \pm 24.20$ & $84.81 \pm 30.39$ & - \\
\hline Aspirin, n (\%) & $16(64.0)$ & $14(26.4)$ & 0.122 \\
\hline $\begin{array}{l}\text { P2Y12 Receptor } \\
\text { Inhibitors, n (\%) }\end{array}$ & $9(36.0)$ & $1(1.9)$ & 0.001 \\
\hline Statins, $\mathrm{n}(\%)$ & $23(92.0)$ & $16(30.2)$ & ¡0.001 \\
\hline Diuretics, n (\%) & $21(84.0)$ & $44(83.0)$ & 0.915 \\
\hline Spironolactone, n (\%) & $18(72.0)$ & $41(77.4)$ & 0.624 \\
\hline ACEI, n (\%) & $14(56.0)$ & $38(71.7)$ & 0.194 \\
\hline $\mathrm{ARB}, \mathrm{n}(\%))$ & $5(20.0)$ & $6(11.3)$ & 0.310 \\
\hline $\beta$-blocker, n (\%) & $24(96.0)$ & $47(88.7)$ & 0.222 \\
\hline Anticoagulant, n (\%) & $4(16.0)$ & $7(13.2)$ & 0.745 \\
\hline Amiodarone, $\mathrm{n}(\%)$ & $2(8.0)$ & $3(5.7)$ & 0.717 \\
\hline $\begin{array}{l}\text { Valsartan Sodium } \\
\text { Tablets, } \mathrm{n}(\%)\end{array}$ & $2(8.0)$ & $1(1.9)$ & 0.304 \\
\hline $\begin{array}{l}\text { Trimetazidine } \\
\text { Hydrochloride, n (\%) }\end{array}$ & $8(32.0)$ & $8(15.1)$ & 0.124 \\
\hline
\end{tabular}

Data are expressed as mean \pm standard deviation or number (\%).

Abbreviations: ACEI, angiotensin-converting enzyme inhibitor; ARB, angiotensin receptor blocker; BNP, B-type natriuretic peptide; CAG, coronary arteriography; CTA, computed tomography angiography; DBP, 
diastolic blood pressure; HBP, high blood pressure; LVEF, left ventricular ejection fraction; NYHA, New York Heart Association; SBP, systolic blood pressure;

\section{All-cause mortality}

The occurrence of all-cause death in ICM group was higher than in DCM group, while SCD and other cardiogenic deaths (including death due to malignant arrhythmia such as ventricular tachycardia, ventricular fibrillation, etc.) were not statistically different between the two groups. Kaplan-Meier multivariate Cox regression analysis showed that ICM group had higher cardiovascular mortality than DCM group, but there was no significant difference of the incidence of cardiopulmonary resuscitation or sustained ventricular tachycardia (VT). (Table 2 )

Table 2. Follow-up results of patients in ICM group and DCM group.

\begin{tabular}{llll}
\hline & ICM $(\mathbf{N}=\mathbf{2 5})$ & DCM $(\mathbf{N}=\mathbf{5 3})$ & P-value \\
\hline Death from any cause & $8(32)$ & $5(9.43)$ & $\mathbf{0 . 0 1 2}$ \\
Cardiovascular death & $5(20)$ & $3(5.66)$ & 0.052 \\
$\begin{array}{l}\text { No cardiovascular } \\
\text { death }\end{array}$ & $3(12)$ & $2(3.77)$ & 0.171 \\
$\begin{array}{l}\text { Resuscitated cardiac } \\
\text { arrest or sustained VT }\end{array}$ & $4(16)$ & $8(15.09)$ & 0.919 \\
$\begin{array}{l}\text { Sustained VT requiring } \\
\text { medical intervention or }\end{array}$ & $2(8)$ & $5(9.43)$ & 0.839 \\
$\begin{array}{l}\text { electrical conversion } \\
\text { Heart transplantation }\end{array}$ & $0(0)$ & & \\
\hline
\end{tabular}

Data are presented as number (\%).

\section{Survival analysis}

Kaplan-Meier survival analysis revealed that the incidence of cardiovascular death during the follow-up period in the ICM group was higher than that in the DCM group $(\mathrm{p}=0.049$; Figure 1$)$. After adjusted for age, LVEF, course of heart failure, NYHA classification, and history of diabetes. Cox regression analysis showed that cardiogenic mortality in ICM group was higher than that in DCM group. (Table 3 )

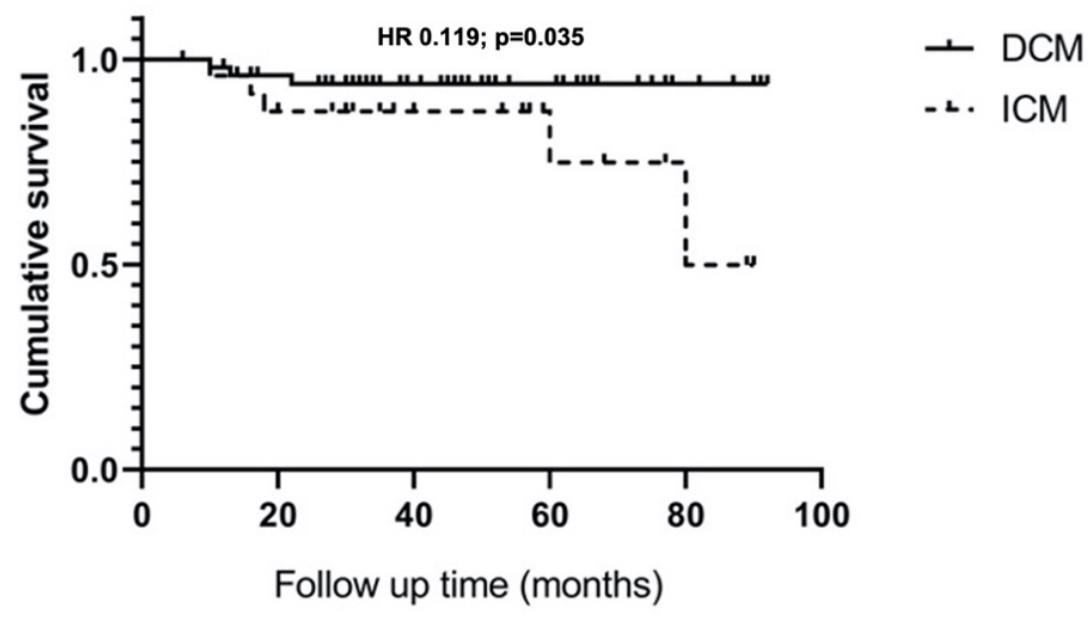


Figure 1:Kaplan-Meier curve analysis of cardiovascular death between two groups.

Table 3.Multivariable Cox regression analysis of cardiovascular death.

\begin{tabular}{llll}
\hline Parameters & HR & $\mathbf{9 5 \% ~ C I ~}$ & P-value \\
\hline DCM vs. ICM & 0.009 & $0.000-0.074$ & $\mathbf{0 . 0 1 8}$ \\
Age & 1.032 & $0.952-1.118$ & 0.445 \\
Sex & 0.937 & $0.074-11.929$ & 0.960 \\
LVEF & 0.728 & $0.578-0.918$ & $\mathbf{0 . 0 0 7}$ \\
Hypertension & 11.526 & $0.928-143.203$ & 0.057 \\
Diabetes & 0.344 & $0.059-2.002$ & 0.235 \\
\hline
\end{tabular}

There was no statistically significant difference in the incidence of cardiopulmonary resuscitation or sustained ventricular tachycardiac ICD discharge in ICM and DCM group $(\mathrm{p}=0.786$; Figure 2$)$. In Cox regression analysis the difference still existed (HR $0.294,95 \%$ CI 0.040 to $2.144, \mathrm{p}=0.227$ ). (Table 4)

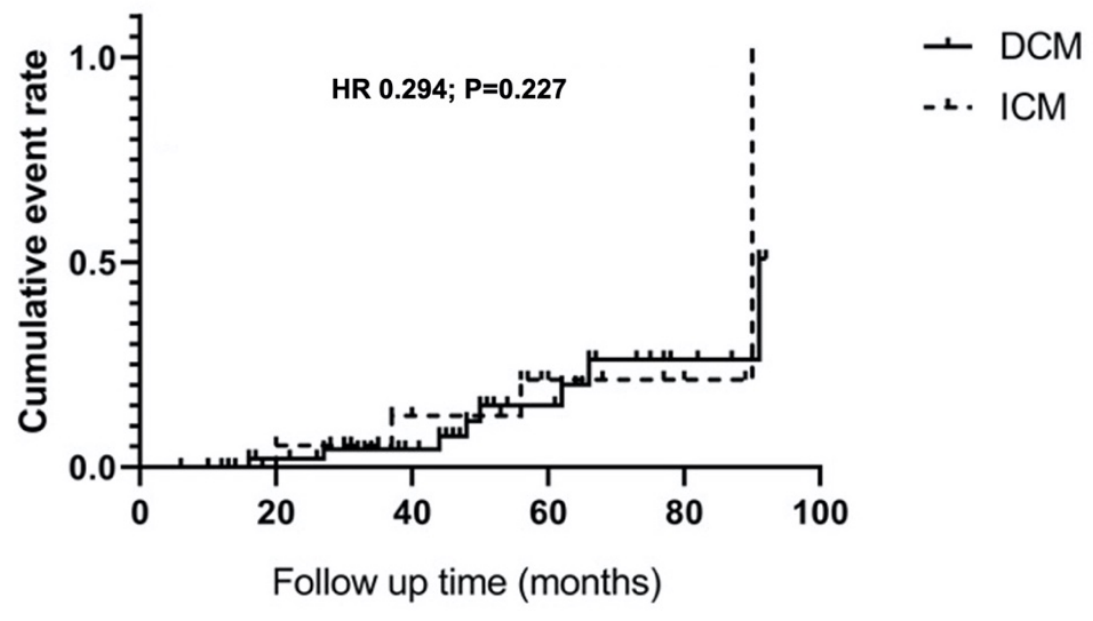

Figure 2:Kaplan-Meier curve analysis of resuscitated cardiac arrest or sustained ventricular tachycardia between two groups.

Table 4. Cox regression analysis of resuscitated cardiac arrest or sustained VT.

\begin{tabular}{llll}
\hline Parameters & HR & $\mathbf{9 5 \% C I}$ & P-value \\
\hline Cause & 0.294 & $0.040-2.144$ & 0.227 \\
Age & 0.086 & $0.975-1.096$ & 0.086 \\
History of HF (months) & 0.989 & $0.973-1.007$ & 0.225 \\
LVEF & 0.975 & $0.866-1.096$ & 0.668 \\
HBP & 2.537 & $0.406-15.840$ & 0.319 \\
Medication & & & \\
B-blocker & 0.494 & $0.049-5.003$ & 0.550 \\
Amiodarone & 0.989 & $0.973-1.007$ & 0.225 \\
\hline
\end{tabular}




\section{Discussion}

In the European Guidelines, ICD implantation is a class 1B recommendation for patients with nonischemic heart failure, and a class $1 \mathrm{~A}$ recommendation for patients with ischemic heart failure. ${ }^{[7]}$ However, no difference was found between the long-term benefits of DCM patients and ICM patients receiving ICD as primary prevention treatment in this study.

Patients with extensive comorbidity may experience less benefit from ICDs than those with less comorbidity. ${ }^{[10]}$ We can see that patients in the ICM group have prevalence of hypertension and diabetes than patients in the DCM group. In this study, $38 \%$ of deaths were attributed to non-cardiovascular diseases, emphasizing the importance of strict screening criteria for ICD implantation. ICD implantation in NICM patients had a less benefit, which provided a basis for not implanting ICD in frail patients, which is in line with the guidelines that recommend. ${ }^{[11,12]}$

Cox regression analysis found that all-cause mortality of ICM was higher than that of DCM group. Patients with more comorbidities benefited less from ICD treatment. Therefore, it is not recommended to install ICDs in patients who cannot tolerate or are not suitable for CRT treatment, VAD (ventricular assist device), NYHA Class IV heart transplantation, or patients with severe comorbidities. The life expectancy of these patients is difficult to exceed One year, it is difficult to obtain benefits from expensive ICD treatment. Studies have also shown that ICM patients in NYHA II-III patients have a greater risk of death than NICM patients, so it is more recommended to carry out drug or ICD primary prevention treatment in the early stage of the disease. ${ }^{[13]}$

As we know, ICD is superior to antiarrhythmic drugs in increasing overall survival. ${ }^{[14]}$ Studies have shown that, ICD was superior to amiodarone for the prevention of SCD. ${ }^{[15]}$ In this study, due to the small number of patients taking amiodarone, and rare arrhythmia events during the follow-up results, so their effects could not be compared.

The European Guidelines have recommended ICD implantation in patients with NYHA class II-III. Although ICD treatment could reduce the risk of SCD in some NYHA class IV patients, these patients often survived less than 1 year due to the presence of severe diabetes, cerebral infarction and other non-cardiovascular diseases. Due to the high survival rate (50\% at 10 years), heart transplantation became a choice for patients with severe HF. ${ }^{[16]}$ However, due to the limited donor heart, these patients had a very high risk of SCD while waiting for surgery, which was a special indication for ICD implantation. Some retrospective studies showed that the use of ICD in patients waiting for heart transplantation can reduce overall mortality by $36 \%$ to $49 \% .{ }^{[17]}$ Interestingly, 2 patients in the DCM group successfully received heart transplantation in this study.

Among the patients included in this study, 3 (12\%) in the ICM group and 20 (37.7\%) in the DCM group have received CRT treatment. ${ }^{[18,19]}$ Reversing LV remodeling is one of the most important effects of CRT. However, it is difficult for CRT to reverse cardiac remodeling of ICM patients whose LV were covered with scar tissues to the same extent as DCM, which was consistent with our results. ${ }^{[20]}$ In this study, more patients received CRT-D treatment in the DCM group, which may be a choice based on the patient's situation in clinical practice, but there is a lack of function evaluation after receiving CRT-D treatment in both groups.

Limitations of our research need to be recognized. The sample size of this study was small, and the conclusions were greatly affected by individual differences. Multi-center studies could be conducted in the future.

In conclusion, DCM patients with ICD implantation compared could be benefit with a reduction in the risk of all-cause mortality and cardiovascular disease with ICM patients, while the occurrence of SCD had no difference in two groups.

\section{Acknowledgements}

This work was supported by grants from the Key Projects of Science and Technology Development Foundation of Nanjing Department of Health (201704003 to L. Wang) and the Six Talent Peaks Project in Jiangsu Province (2016-WSN-157 to L. Wang). 


\section{Declarations}

The authors declare no conflict of interest.

\section{References}

1. Tayal U, Prasad S and Cook SA. Genetics and genomics of dilated cardiomyopathy and systolic heart failure. Genome Med . 2017;9:20.

2. Thomas T, Nancy, Haase,Wayne et al . Heart disease and stroke statistics-2006 update: a report from the American Heart Association Statistics Committee and Stroke Statistics Subcommittee.Circulation . 2006;113:85-151.

3. Halliday BP, Cleland JGF, Goldberger JJ et al . Personalizing Risk Stratification for Sudden Death in Dilated Cardiomyopathy: The Past, Present, and Future. Circulation . 2017;136:215-231.

4. Sun WP, Li CL, Guo JC et al . Long-term efficacy of implantable cardiac resynchronization therapy plus defibrillator for primary prevention of sudden cardiac death in patients with mild heart failure: an updated meta-analysis. Heart Fail Rev . 2016;21:447-53.

5. Pathak RK, Sanders P and Deo R. Primary prevention implantable cardioverter-defibrillator and opportunities for sudden cardiac death risk assessment in non-ischaemic cardiomyopathy. Eur Heart $J$. 2018;39:2859-2866.

6. Kolodziejczak M, Andreotti F, Kowalewski M et al . Implantable Cardioverter-Defibrillators for Primary Prevention in Patients With Ischemic or Nonischemic Cardiomyopathy: A Systematic Review and Metaanalysis. Ann Intern Med . 2017;167:103-111.

7. Ponikowski P, Voors AA, Anker SD et al . 2016 ESC Guidelines for the diagnosis and treatment of acute and chronic heart failure: The Task Force for the diagnosis and treatment of acute and chronic heart failure of the European Society of Cardiology (ESC)Developed with the special contribution of the Heart Failure Association (HFA) of the ESC.Eur Heart J . 2016;37:2129-2200.

8. Priori SG, Blomstrom-Lundqvist C, Mazzanti A et al , Task Force for the Management of Patients with Ventricular A and the Prevention of Sudden Cardiac Death of the European Society of C. 2015 ESC Guidelines for the management of patients with ventricular arrhythmias and the prevention of sudden cardiac death: The Task Force for the Management of Patients with Ventricular Arrhythmias and the Prevention of Sudden Cardiac Death of the European Society of Cardiology (ESC)Endorsed by: Association for European Paediatric and Congenital Cardiology (AEPC).Europace . 2015;17:1601-87.

9. Bansch D, Antz M, Boczor S et al . Primary prevention of sudden cardiac death in idiopathic dilated cardiomyopathy: the Cardiomyopathy Trial (CAT). Circulation . 2002;105:1453-8.

10. Steinberg BA, Al-Khatib SM, Edwards R et al . Outcomes of implantable cardioverter-defibrillator use in patients with comorbidities: results from a combined analysis of 4 randomized clinical trials. JACC Heart Fail . 2014;2:623-9.

11. Kober L, Thune JJ, Nielsen JC et al . Defibrillator Implantation in Patients with Nonischemic Systolic Heart Failure. N Engl J Med . 2016;375:1221-30.

12. Sticherling BASMSKDBSOC. Application of a mortality risk score in a general population of patients with an implantable cardioverter defibrillator (ICD). Heart . 2014;100:487-491.

13. Barsheshet A, Moss AJ, Huang DT et al . Applicability of a risk score for prediction of the long-term (8-year) benefit of the implantable cardioverter-defibrillator. J Am Coll Cardiol . 2012;59:2075-9.

14. Investigators TAvIDA. A comparison of antiarrhythmic-drug therapy with implantable defibrillators in patients resuscitated from near-fatal ventricular arrhythmias. The Antiarrhythmics versus Implantable Defibrillators (AVID) Investigators. The New England journal of medicine . 1997;337:1576-1584. 
15. Hjalmarson A GS, Fagerberg B, Wedel H et al. Effects of controlled-release metoprolol on total mortality, hospitalizations, and well-being in patients with heart failure: the Metoprolol CR/XL Randomized Intervention Trial in congestive heart failure (MERIT-HF). MERIT-HF Study Group. JAMA . 2000;283:1295-1302.

16. Saba S, Atiga WL, Barrington W et al . Selected patients listed for cardiac transplantation may benefit from defibrillator implantation regardless of an established indication. The Journal of Heart and Lung Transplantation . 2003;22:411-418.

17. Ermis C ZG, Zhu AX, Fabian W et al . Improved survival of cardiac transplantation candidates with implantable cardioverter defibrillator therapy: role of beta-blocker or amiodarone treatment.J Cardiovasc Electrophysiol . 2003;14:1540-1546.

18. Wells G PR, Healey JS, Talajic M et al . Cardiac resynchronization therapy: a meta-analysis of randomized controlled trials. Canadian Medical Association Journal . 2011;183:421-429.

19. Bilchick KC, Kamath S, DiMarco JP et al. Bundle-branch block morphology and other predictors of outcome after cardiac resynchronization therapy in Medicare patients. Circulation . 2010;122:2022-30.

20. Sohaib SM, Finegold JA, Nijjer SS et al . Opportunity to increase life span in narrow QRS cardiac resynchronization therapy recipients by deactivating ventricular pacing: evidence from randomized controlled trials. JACC Heart Fail . 2015;3:327-36. 